\title{
Serotonin triggers swarming in locusts
}

Desert locusts (Schistocerca gregaria) are normally solitary insects, but in crowded conditions, they undergo physical and behavioral changes and become 'gregarious', clustering in swarms that can include billions of individuals. These swarms devastate crops over large areas of land, causing economic hardship and affecting the livelihood of as many as one in ten people worldwide. The locusts' transition from solitary to gregarious is triggered by sensory stimuli: either jostling from or the sight and smell of other locusts nearby. Until recently, however, the mechanism of action of these stimuli was not known. Now, scientists report that an increase in serotonin concentration in a specific region of the locust's nervous system underlies the behavioral changes that lead to swarming.

The scientists, headed by Michael Anstey (University of Oxford, UK) and Stephen M. Rogers (University of Cambridge, UK), began by showing that solitary locusts could become fully gregarious within $2 \mathrm{~h}$ of forced crowding and that this 2 -h period

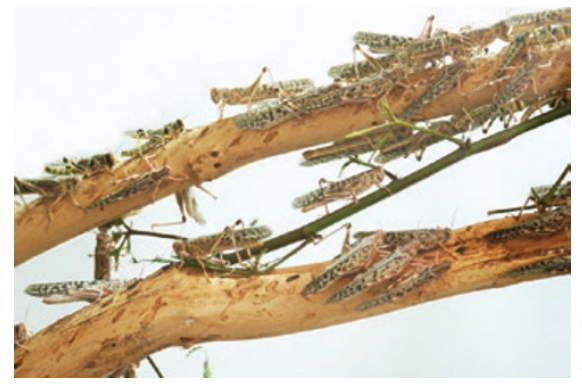

also featured an increase in serotonin concentration in the thoracic ganglia, but not in the brain or other regions of the nervous system (Science 323, 627-630; 2009). Using a series of experiments, the researchers set out to establish whether the increase in serotonin was the causative factor for the behavior change. First, they analyzed the degree of gregariousness in relation to the amount of serotonin in locusts crowded for various lengths of time. They found that serotonin concentration was positively correlated with the degree of gregariousness. Next, they assessed the effects on locust behavior of blocking either the action or the production of serotonin and found that either block prevented locusts from becoming gregarious. Then, they evaluated whether administering serotonin or serotonin agonists would induce locusts to become gregarious, even in the absence of sensory stimuli associated with other locusts, and found that locusts treated with the compounds were more gregarious than untreated controls. Finally, they asked whether boosting the locusts' natural synthesis of serotonin would encourage them to become more gregarious when exposed to sensory stimuli from other locusts and found that the treatment did enhance gregariousness.

The combined results of the experiments show that an increase in serotonin concentration in the thoracic ganglia of locusts is both necessary and sufficient to trigger the transformation from solitary to gregarious. The identification of this trigger may facilitate development of methods of suppressing or inhibiting serotonin in order to control swarming in locusts.

Monica Harrington

\section{A CANCER-EIGHTING IMPLANT?}

Cancer is often associated with a weak immune response, so many approaches to treating the disease involve stimulation of immune cells called dendritic cells. When dendritic cells encounter tumor (or other) antigens, they process them and present them to naive T cells. This activates the T cells and 'teaches' them to identify and attack tumor cells. In most cell-based vaccination treatments, dendritic cells are isolated from the patient, activated ex vivo and then re-introduced into the patient. So far, the efficacy of this treatment has been limited; more than $90 \%$ of dendritic cells die after transplantation, and few make it to the lymph nodes, where the T cells reside. Furthermore, the treatment is costly and cumbersome, requiring two patient procedures.

Now, a study led by David J. Mooney (Harvard University and Wyss Institute for Biologically Inspired Engineering, Cambridge, MA) suggests that an effective cancer vaccine that works directly in the body may be within reach. The researchers developed porous, biodegradable polymer 'scaffolds': small, sponge-like discs that can be filled with materials and then implanted in the body, where the materials are gradually released. In a series of experiments, the investigators loaded the scaffolds with a protein known to stimulate recruitment and proliferation of dendritic cells (Nat. Mater. 8, 151-158; 2009). Recruited dendritic cells entered the scaffold's pores, where they were activated and housed until release. To test the structure's potential as a cancer vaccine, Mooney and colleagues added melanoma tumor lysates to the scaffold so that dendritic cells that entered the structure would be loaded with tumor antigens.

The researchers implanted these structures into subcutaneous pockets in the backs of mice. Two weeks later, they injected the implanted mice with highly aggressive and metastatic melanoma cells. All mice that were implanted with 'empty' scaffolds developed tumors within 18 days and had to be euthanized shortly afterward. Mice that were implanted with scaffolds containing antigen alone fared somewhat better, though all died by day 40. In mice that were implanted with scaffolds containing both tumor antigen and the dendritic cell-stimulating protein, tumor formation was delayed by approximately 40 days in $50 \%$ of the mice, and $23 \%$ of the mice were cured.

This study could have profound implications for the development of cancer vaccines and treatments for other diseases. The scaffolds are made of materials that are safe for humans, so clinical testing may be possible in the near future.

Karen Marron 